\title{
Helicobacter pylori infection is an independent risk factor for Runx3 methylation in gastric cancer
}

\author{
YOSHIHIKO KITAJIMA, KAZUMA OHTAKA, MAYUMI MITSUNO, \\ MASAYUKI TANAKA, SEIJI SATO, YUJI NAKAFUSA and KOHJI MIYAZAKI \\ Department of Surgery, Saga University Faculty of Medicine, 5-1-1 Nabeshima, Saga 849-8501, Japan
}

Received September 28, 2007; Accepted November 5, 2007

\begin{abstract}
Runx3, a member of the human runt-related transcription factor family, is known as a possible tumor suppressor gene for gastric cancer. Runx 3 expression is frequently suppressed by the promoter hypermethylation in gastric cancer cell lines and tissues. However, the precise mechanism of the induction of Runx 3 methylation, which is considered to be a critical step in gastric carcinogenesis, remains to be elucidated. In the present study, we evaluated runx 3 gene methylation in 57 resected early gastric cancer specimens. Then, we correlated Runx3 methylation in the cancer tissue specimens with clinicopathological factors as well as the mucosal backgrounds, such as intestinal metaplasia surrounding the cancer cells and Helicobacter pylori $(H$. pylori) infection. Runx 3 methylation was observed in 30 of the $57(52.6 \%)$ cancer specimens, whereas methylation was detected in 10 of the $57(17.5 \%)$ corresponding noncancerous mucosae. In comparison to the clinicopathological factors, Runx 3 methylation was significantly correlated with both age and tumor location. A multivariate analysis demonstrated that age and tumor location as well as $H$.pylori infection were independent risk factors for Runx3 methylation. We demonstrated for the first time that $H$. pylori infection contributes to Runx3 methylation in gastric cancer tissues. When a persistent infection by $H$. pylori continues in the middle/lower stomach for a long period, Runx3 methylation may be induced and the subsequent loss of Runx3 expression may therefore affect gastric carcinogenesis.
\end{abstract}

\section{Introduction}

The human runt-related transcription factors (Runx) are important targets of transforming growth factor (TGF)- $\beta$ superfamily signaling (1). The Runx genes runx 1, runx2 and

Correspondence to: Dr Yoshihiko Kitajima, Department of Surgery, Saga University Faculty of Medicine, 5-1-1 Nabeshima, Saga 849-8501, Japan

E-mail: kitajiy@cc.saga-u.ac.jp

Key words: Runx3, methylation, Helicobacter pylori, gastric cancer runx3 have been identified as human homologs of the Drosophila genes runt (1). In a recent study, the gastric mucosa of a Runx3 knockout mouse exhibited hyperplasia due to stimulated proliferation and suppressed apoptosis, which was accompanied by reduced sensitivity to TGF- 3 (2). This finding identified Runx3 as a tumor suppressor gene for genesis of gastric cancer. In addition, intestinal metaplasia (IM) is generally regarded as a precancerous gastric lesion for intestinal type gastric cancer (3). In the Runx3 knockout mouse gastric epithelial cells, some cells differentiated into intestinal type cells suggesting that the loss of Runx3 expression triggered precancerous IM, which possibly leads to cancer development in the stomach (4).

In gastric cancer cell lines and tissues, the promoter region of the Runx 3 gene is frequently methylated and such methylation is correlated with the loss of Runx 3 expression (5). Runx3 methylation has also been found in non-cancerous diseases such as chronic gastritis, IM and gastric adenoma with less frequency than cancer (6). These studies demonstrate one possible carcinogenic pathway, namely that the loss of Runx3 expression by promoter methylation causes chronic gastritis or IM in gastric mucosa, which leads to the development of gastric cancer.

Helicobacter pylori (H. pylori) infection is a major causative factor for gastric cancer, chronic gastritis, peptic ulcers, atrophic gastritis and IM (7-9). The risk of patients with $H$. pylori infection developing gastric cancer is in the order of two- to six-fold according to most retrospective casecontrol and prospective epidemiologic studies (7). Several factors have been reported which affect gastric carcinogenesis by $H$. pylori infection $(8,9)$. For instance, the cytotoxin-associated gene (cag) of $H$. pylori is one of the most studied virulences and has been reported to have oncogenic effects $(8,10)$. On the other hand, several current reports have demonstrated that high levels of aberrant methylation of several $\mathrm{CpG}$ islands were isolated in $H$. pylori infected gastric mucosae and its possible association with gastric cancer risk (11). However, whether or not $H$. pylori infection directly induces Runx 3 methylation in the gastric mucosa remains to be elucidated.

In the present study, we investigated Runx3 methylation in 57 early gastric cancer tissues and corresponding noncancerous tissues. The existence of IM was histologically determined on adjacent mucosa surrounding the cancer cells. $\mathrm{H}$. pylori infection in the samples was assessed by PCR of the 16S rRNA and cagA region in H. pylori DNA according to a 
previous study (12). The methylation status of Runx3 was compared with clinicopathological factors and the mucosal background such as IM or H. pylori infection. The aim of this study was to clarify whether or not $H$. pylori infection directly affects IM or Runx3 methylation in gastric cancer tissues.

\section{Materials and methods}

Patients and tissue samples. Fifty-seven patients with early gastric cancer, who underwent gastrectomy from March 2001 to July 2006 at Saga University Hospital, were eligible for the present study. The patients gave informed consent for specimen collection, which is required by the Ethics Committee. All of the specimens were examined macroscopically and histologically based on criteria proposed by the Japanease Research Society for Gastric Cancer (13). Intestinal metaplasia (IM) on the 57 cancer specimens was histologically assessed by two pathologists (D.M. and K.K.). Samples from cancer tissues and the corresponding noncancerous mucosa were freshly obtained from the resected stomach and stored at $-80^{\circ} \mathrm{C}$ until use. DNA was purified using the EZ1 DNA tissue kit in BioRobot ${ }^{\circledR} \mathrm{EZ1}$ workstation (Qiagen, GmbH Hilden, Germany).

Bisulfite modification and methylation-specific PCR (MSP). Treatment of DNA samples with bisulfite converts all unmethylated cytosines to uracils, while leaving the methylcytosines unaltered. This allows for the subsequent differentiation of methylated and unmethylated sequences by methylation-specific PCR (MSP). Bisulfite modification of DNA $(1 \mu \mathrm{g})$ was carried out according to the method described previously (14). Then, $30 \mu 1$ of the modified DNA solution was obtained and $1 \mu 1$ of $30 \mu 1$ was subjected to MSP. The methylated (M) and unmethylated (U)-specific primers for Runx3 were synthesized according to a previous report (15). M-specific primers: 5'-TTCGTTTATTTTGTCGTCG TCGT-3' (forward), 5'-CGCTATTATACGTATTCCCG-3' (reverse). U-specific primers: 5'-TTTGGGTTTATGGGAAT ATG-3' (forward), 5'-TTCTACAACAACAACAACC-3' (reverse). PCR was performed using the exTaq polymerase system (Takara, Tokyo, Japan) under the following conditions. One cycle at $96^{\circ} \mathrm{C}$ for $3 \mathrm{~min}, 35$ cycles at $96^{\circ} \mathrm{C}$ for $30 \mathrm{sec}, 55^{\circ} \mathrm{C}$ for $30 \mathrm{sec}, 72^{\circ} \mathrm{C}$ for $30 \mathrm{sec}$ and 1 cycle at $72^{\circ} \mathrm{C}$ for $5 \mathrm{~min}$. An artificial methylated DNA (universal methylated DNA, Invitrogen, Purchase, NY, USA) and an unmethylated DNA isolated from normal lymphocyte were used for the methylation- and unmethylation-specific reactions, respectively. Distilled water without DNA was used as a negative control. PCR products were analyzed in $1.5 \%$ agarose gel and stained with ethidium bromide. The proposed size of the PCR product from the methylationspecific primer is $100 \mathrm{bp}$, whereas that from the unmethylation primer is $120 \mathrm{bp}$.

PCR detection of $H$. pylori infection. DNA of $H$. pylori on both of the cancer and the corresponding non-cancerous tissues was amplified using PCR. The primers were designed for the 16 rDNA and cagA region in the $H$. pylori genome as previously reported (12). 16r DNA-specific primers: 5'-TGC GAAGTGGAGCCAATCTT-3' (forward), 5'-GGAACGTA
Table I. Relationship between Runx3 methylation and clinicopathological factors.

\begin{tabular}{|c|c|c|c|}
\hline & \multicolumn{2}{|c|}{ Runx3 } & \multirow[b]{2}{*}{ P-value } \\
\hline & $\mathrm{U}$ & M & \\
\hline Age & $62.5 \pm 2.32$ & $68.6 \pm 1.82$ & 0.04 \\
\hline \multicolumn{4}{|l|}{ Gender } \\
\hline $\mathrm{M}$ & 14 & 20 & \\
\hline $\mathrm{F}$ & 13 & 10 & 0.26 \\
\hline \multicolumn{4}{|l|}{ Location } \\
\hline $\mathrm{U}$ & 8 & 1 & \\
\hline $\mathrm{M} / \mathrm{L}$ & 19 & 29 & 0.01 \\
\hline \multicolumn{4}{|c|}{ Histology } \\
\hline Diff & 16 & 16 & \\
\hline Undiff & 11 & 14 & 0.65 \\
\hline \multicolumn{4}{|l|}{$\mathrm{T}$} \\
\hline $\mathrm{m}$ & 14 & 11 & \\
\hline $\mathrm{sm}$ & 13 & 19 & 0.25 \\
\hline \multicolumn{4}{|l|}{$\mathrm{N}$} \\
\hline- & 24 & 25 & \\
\hline+ & 3 & 5 & 0.71 \\
\hline \multicolumn{4}{|l|}{ ly } \\
\hline- & 24 & 22 & \\
\hline+ & 3 & 8 & 0.19 \\
\hline \multicolumn{4}{|l|}{$\mathrm{V}$} \\
\hline- & 25 & 30 & \\
\hline+ & 2 & 0 & 0.22 \\
\hline
\end{tabular}

U, upper stomach; M/L, middle/lower stomach; Diff, differenetiated carcinoma; Undiff, undifferentiated carcinoma; $\mathrm{T}$, depth of cancer invasion; $\mathrm{N}$, lymph node metastasis; ly, invasion to lymphatic vessel; $\mathrm{v}$, invasion to venus vessel.

TTCACCGCAACA-3' (reverse). CagA-East Asian-specific primers: 5'-AAAGGAGTGGGCGGTTTCA-3' (forward), 5'-CCTGCTTGATTTGCCTCATCA-3' (reverse). The experimental conditions for PCR were the same as for the MSP analysis. The proposed sizes were $118 \mathrm{bp}$ for $16 \mathrm{~S}$ rRNA and $91 \mathrm{bp}$ for CagA-East Asian, respectively.

Statistical analysis. The statistical analysis was performed using the $\chi^{2}$ test and the Mann-Whitney U test. The student's t-test was used to determine group differences. For all statistical analyses, the StatView ${ }^{\circledR} 5.0$ software program (SAS Institute, Cary, USA) was used. Multivariate logistic regression analysis was used to analyze the risk factor for Runx3 methylation. Statistical significance was established at a value of $\mathrm{P}<0.05$.

\section{Results}

MSP analysis of the runx3 gene in early gastric cancer tissues. Fifty-seven paired DNA from early gastric cancer and the 

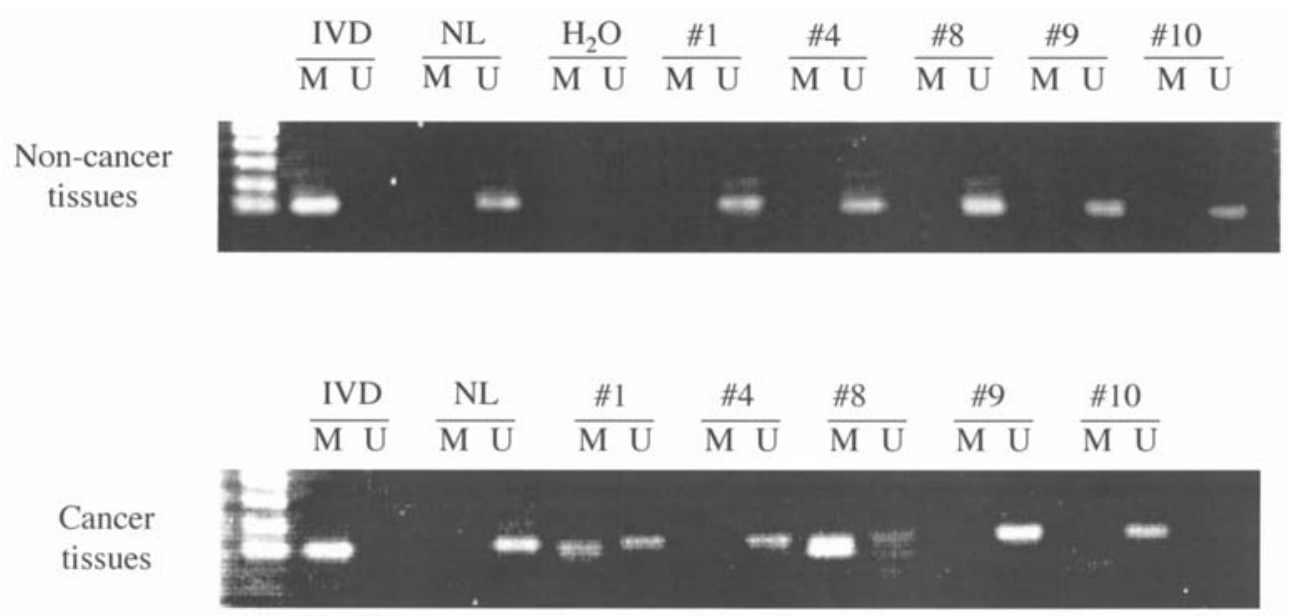

Figure 1. An MSP analysis of the runx3 gene using 5 paired cancer and corresponding non-cancerous tissues (\#1, \#4, \#8, \#9 and \#10). IVD: in vitro methylated DNA NL: DNA from normal lymphocyte. M, methylated; U, unmethylated.
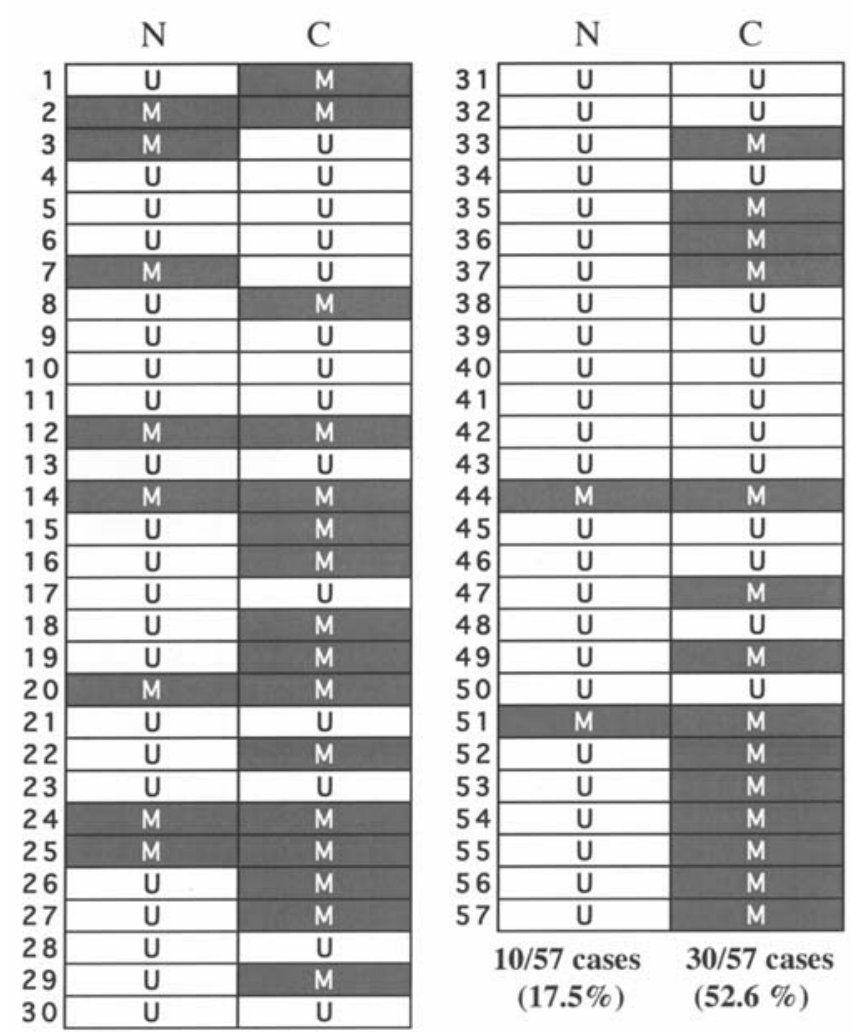

Figure 2. Summarized results of an MSP analysis using the 57 gastric cancer tissues and the corresponding non-cancerous mucosa. $\mathrm{N}$, non-cancerous tissues; C, cancer tissues; M, methylated; U, unmethylated.

corresponding non-cancerous mucosa were treated with bisulfite and subjected to methylation-specific PCR (MSP) for detecting runx3 gene methylation. As shown in Fig. 1, the PCR product at $100 \mathrm{bp}$ from the methylation-specific primer was observed in a positive control IVD, whereas the DNA fragment at $120 \mathrm{bp}$ length from unmethylation-specific primers was found in the normal lymphocyte (NL). No signal from the water sample was detected. In all 5 samples from the non-cancerous mucosa, there were unmethylation- and nonmethylation-specific signals. In contrast, methylation-specific
Table II. PCR detection of 16S rRNA and cagA from $H$. pylori DNA in gastric mucosae.

\begin{tabular}{|c|c|c|c|c|c|c|}
\hline & \multicolumn{3}{|c|}{ Non-cancerous tissues } & \multicolumn{3}{|c|}{ Cancer tissues } \\
\hline & \multicolumn{3}{|c|}{ 16S rRNA } & \multicolumn{3}{|c|}{ 16S rRNA } \\
\hline & - & + & $\mathrm{P}$ & - & + & $\mathrm{P}$ \\
\hline \multicolumn{7}{|c|}{$\operatorname{cag} \mathrm{A}$} \\
\hline- & 15 & 14 & & 17 & 22 & \\
\hline+ & 3 & 25 & 0.001 & 0 & 18 & $<0.001$ \\
\hline
\end{tabular}

signals were found in cases 1 and 8 from the cancer specimens. A summary of the result of MSP is shown in Fig. 2. Thirty of the 57 cancer tissues $(52.6 \%)$ were assessed as methylation positive, while 10 of the $57(17.5 \%)$ normal mucosae were also assessed as methylated. Of the 10 noncancerous mucosa with Runx3 methylation, cases 3 and 7 did not show the methylation in the corresponding cancer specimens.

Relationship between Runx3 methylation and clinicopathological factors. Table I shows the relationship between Runx3 methylation in the 57 cancer tissues and the clinicopathological factors. Runx3 methylation was significantly correlated with age and tumor location, whereas the methylation did not show correlation with gender, depth of cancer invasion (T), histological type, lymph node metastases (N) and vessel invasion (ly and v).

Relationship between H. pylori infection and Runx3 methylation. We assessed $H$. pylori infection on the 57 paired samples by PCR of $16 \mathrm{~S}$ rRNA and the cagA region in $H$. pylori DNA. Fig. 3 shows the PCR results of $16 \mathrm{~S}$ rRNA and East-Asian cagA in 5 gastric cancer tissues. 16S rRNA at 118 bp length were observed in 4 of the 5 DNA samples, whereas the products at $91 \mathrm{bp}$ from East-Asian cagA were 2 of the 5 samples. As summarized in Table II, DNA from 16S rRNA was amplified in 39 of the 57 (68.4\%) non-cancerous 

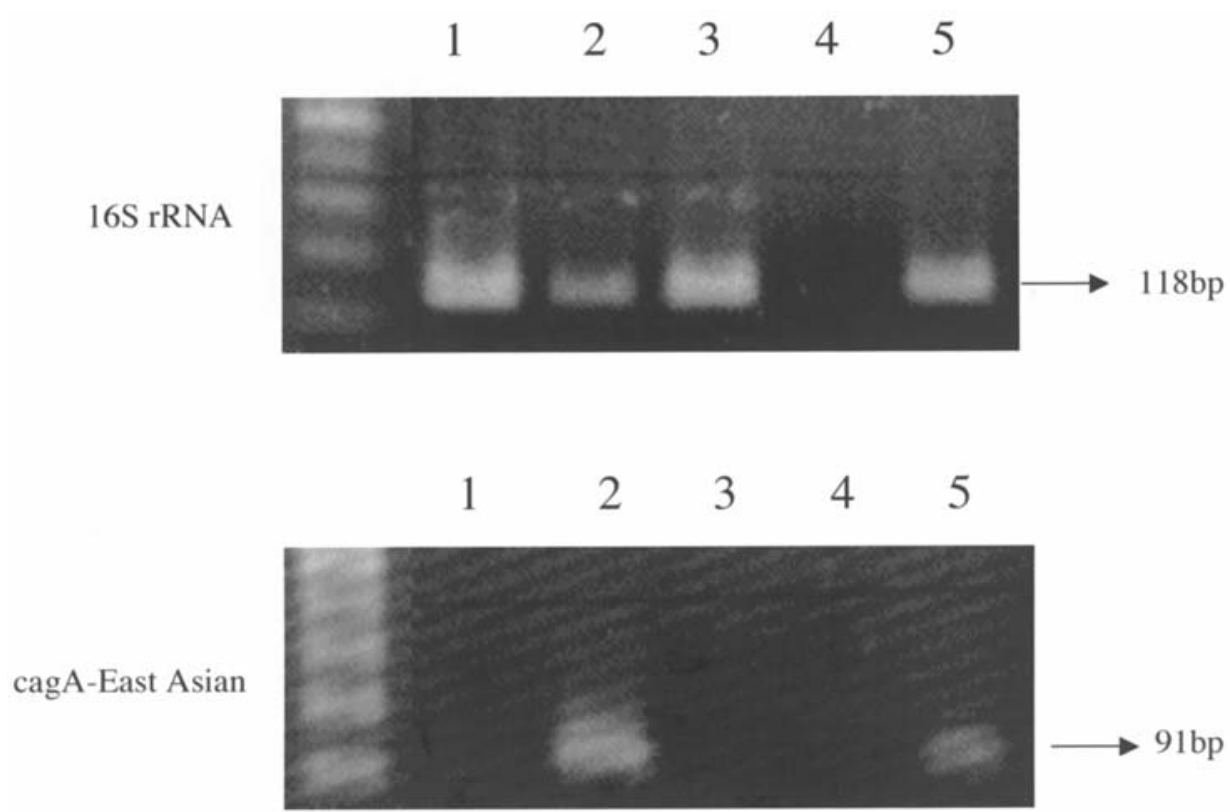

Figure 3. PCR amplification of $16 \mathrm{~S}$ rRNA and East-Asian cagA in H. pylori DNA. Asterisk: positive signal. Arrows: PCR products from 16S rRNA at 118 bp and cag A at 91 bp length.

Table III. Relationship between Runx3 methylation and mucosal background.

\begin{tabular}{lrrr}
\hline & \multicolumn{2}{c}{ Runx-3 } & \\
\cline { 2 - 3 } & $\mathrm{U}$ & $\mathrm{M}$ & $\mathrm{P}$ \\
\hline $\mathrm{IM}$ & & & \\
- & 10 & 4 & \\
+ & 17 & 26 & 0.06 \\
H. pylori infection & & & \\
16S rRNA & & & \\
- & 11 & 5 & \\
+ & 16 & 24 & 0.09 \\
CagA & & & \\
- & 19 & 20 & \\
+ & 8 & 10 & 0.76 \\
\hline
\end{tabular}

IM, intestinal metaplasia.

mucosae while that from East-Asian cagA was 28 of the 57 $(49.1 \%)$. These two factors showed significant correlation $(\mathrm{p}=0.001)$. Similarly, $16 \mathrm{~S}$ rRNA East-Asian cagA were detected in 40 of the $57(70.2 \%)$ and 18 of the $57(31.6 \%)$ cancer tissues, respectively. There was a strong correlation between 16rDNA and cagA in the 57 cancer tissues $(\mathrm{p}<0.001)$.

Analysis of the risk factor for Runx3 methylation. In order to clarify whether or not mucosal background affects Runx3 methylation, the relationship between the methylation status in the cancer tissues and IM, H. pylori infection was analyzed (Table III). Although no statistical significance was found, Runx 3 methylation showed a trend of positive
Table IV. Multivariate logistic regression analysis for the risk factor affecting Runx-3 methylation in cancer tissues.

\begin{tabular}{lccl}
\hline & Risk ratio & $95 \%$ CI & P-value \\
\hline Age & 6.04 & $0.84-0.98$ & 0.014 \\
Location & 8.26 & $3.21-477$ & 0.004 \\
IM & 0.11 & $0.13-4.26$ & 0.74 \\
16S rRNA & 8.37 & $2.27-70.7$ & 0.004 \\
\hline
\end{tabular}

CI, confidence interval.

correlation with the existence of IM $(\mathrm{p}=0.06)$ and 16S rRNA $(\mathrm{p}=0.09)$. We then performed a multivariate analysis using logistic regression analysis to isolate the risk factors for Runx3 methylation (Table III). As a result, age, tumor location and 16S rRNA were independent risk factors for Runx3 methylation in the cancer tissues.

\section{Discussion}

Gastric cancer is one of the most common malignancies in the world. Various studies on oncogenes, tumor suppressor genes, cell adhesion molecules and cell cycle regulators have been performed in order to clarify the carcinogenesis of gastric cancer (3). Among these studies, one notable experiment using knockout mice indicated the runx3 gene as a possible tumor suppressor gene of gastric cancer (2). The aberrant methylation of the runx3 gene, which is the main mechanism of deficient Runx3 expression in gastric cancer tissues, has been studied in various literature $(5,6)$. Moreover, the aberrant methylation of Runx 3 in non-cancerous gastric 
mucosa has been reported (6). Kim et al reported that Runx3 methylation was found in $64 \%$ of gastric cancer, $8 \%$ of chronic gastritis, $28 \%$ of IM and $27 \%$ of gastric adenoma (6). These results suggest that Runx 3 is a target for epigenetic gene silencing in gastric carcinogenesis and is proposed to play an important role in the carcinogenic sequence from the precancerous state to carcinoma (6). However, the precise mechanism by which Runx 3 methylation occurs in carcinoma cells or non-cancerous gastric mucosa remains to be determined.

In the present study, we analyzed the aberrant methylation of Runx 3 in 57 early gastric cancers and the corresponding non-cancerous mucosa. Furthermore, the mucosal background such as IM and $H$. pylori infection in the 57 gastric cancer tissues was investigated and correlated with the methylation status. In particular, we focused on the critical issues that H. pylori infection, diagnosed by the PCR method, contributes to Runx3 methylation in gastric cancer cells. As a result, Runx3 methylation was frequently observed in 37 of the 57 (52.6\%) cancer tissues in comparison to 10 of the 57 (17.5\%) non-cancerous tissues (Fig. 2). In a comparative analysis of the clinicopathological factors, Runx 3 methylation in the cancer tissues did not correlate with the depth of cancer invasion, lymph node metastasis or vessel invasion, indicating the involvement of Runx3 in gastric carcinogenesis. On the contrary, age and cancer location were both significantly correlated with methylation. Previous studies have demonstrated a significant correlation between aberrant gene methylation in non-cancerous gastric mucosa and aging $(16,17)$. Kang et al demonstrated using gastric epithelium with chronic gastritis that 5 of the 11 genes showed a general increase in the methylation frequency as a function of aging (17). Our study might support this link of aging to Runx3 methylation, although we did not find any significant correlation in the non-cancerous tissues. Runx3 methylation in the 57 cancer tissues was more frequently found in the middle/lower region of the stomach than the upper region. We speculated that the difference in the methylation frequency might depend on the mucosal background surrounding the cancer cells. To study this, we analyzed the association between IM and Runx3 methylation in the 57 cancer tissues. As shown in Table III, IM exhibited the trend of a positive correlation with Runx 3 methylation in the cancer tissues, although there was no statistical significance $(\mathrm{p}=0.06)$. The relationship between IM and cancer location was also assessed and revealed a statistically significant correlation ( $\mathrm{p}=0.03$ ) (data not shown). These results indicated that IM occurs more frequently in the $\mathrm{M} / \mathrm{L}$ region of the stomach and also that Runx3 methylation may affect IM formation surrounding cancer cells.

$H$. pylori infection is known as an important aetiological risk factor in gastric cancer and has been classified as a group I or defined carcinogen by the World Health Organization's International Agency for Research on Cancer (18). H. pylori infection causes chronic active inflammation in the gastric mucosa and may cause mucosal atrophy and intestinal metaplasia (IM), which are known as risk factors for gastric cancer (9). Uemura et al followed up 1,526 Japanese patients with upper gastrointestinal diseases for 7.8 years and observed that gastric cancer developed only in $H$. pylori infected patients, especially those with severe gastric atrophy and intestinal metaplasia (IM) (9). These studies prompted a possible scenario that $H$. pylori infection in gastric mucosa initiates Runx3 methylation, followed by IM formation and leads to cancer development. No study has yet revealed the critical attribution of $H$. pylori infection to Runx 3 methylation in gastric cancer. $H$. pylori infection is usually diagnosed by a histological examination, a serum anti- $H$. pylori IgG antibody test, rapid urease test or culture test (8). Recently, it has been reported that the PCR method developed a new diagnostic tool to detect the $H$. pylori gene with both high sensitivity and specificity $(12,19)$. In the present study, we thus assessed $H$. pylori infection in the cancer as well as the non-cancerous mucosa by PCR amplification of $16 \mathrm{~S}$ rRNA and the East-Asian cagA region as described (12). As shown in Table II, 16S rRNA was highly detected in the normal (68.4\%) and cancer (70.2\%) tissues. East-Asian cagA DNA was also amplified in both the normal $(49.1 \%)$ and cancer tissues $(31.6 \%)$. A statistical analysis showed a strong correlation between 16S rRNA and East-Asian cagA in the normal and cancer tissues. These results indicate that $H$. pylori are frequently infected in gastric cancer tissues and the strains were considerably integrated by East-Asian cagA. Using this highly sensitive method, we revealed that $H$. pylori infection, which was estimated by $16 \mathrm{~S}$ rRNA, showed a higher tendency of Runx3 methylation $(\mathrm{p}=0.09)$. In the multivariate analysis, we demonstrated that $H$. pylori infection was one of the independent risk factors for runx-3 methylation. Several studies have reported similar aspects that aberrant DNA methylation in gastric mucosa is induced by $H$. pylori infection $(11,20)$. Maekita et al quantitatively estimated the methylation levels of $8 \mathrm{CpG}$ islands (CGIs) in gastric mucosa, which were collected from healthy volunteers with or without $H$. pylori infection (11). The results demonstrated that the methylation levels of all of the 8 CGI regions were significantly higher in the $H$. pylori positive than in the $H$. pylori negative mucosa. Chan et al showed a significant relationship between the methylation of E-cadherin and H. pylori infection in gastric mucosa from dyspeptic patients, indicating that the promoter methylation of E-cadherin associated with $H$. pylori infection occurs as an early event in gastric carcinogenesis (20). These findings might support our notion that runx-3 methylation is induced by $H$. pylori infection and the subsequent silencing of runx-3 expression may correlate with gastric carcinogenesis. The cagA protein, which is encoded by the cagA gene is a highly immunogenic protein and is one of the most studied virulence factors of $H$. pylori $(8,21,22)$. In the present analysis, cagA appeared not to affect Runx3 methylation (Table III). An alternative mechanism other than cagA may induce Runx 3 methylation in $H$. pylori-infected gastric mucosa.

In conclusion, we demonstrated for the first time that $H$. pylori infection is an independent risk factor for aberrant methylation of the runx 3 gene in gastric cancer. A multivariate analysis also revealed that age and cancer location were independent factors for Runx 3 methylation. H. pylori infection persisting for many years may induce Runx3 methylation in the epithelial cells at the $\mathrm{M} / \mathrm{L}$ region of the stomach. However, we did not observe any correlation beween Runx3 methylation and $H$. pylori infection in the 
non-cancerous mucosae. It is therefore plausible that some molecular alteration may be additionally necessary for developing gastric cancer in non-cancerous mucosa with Runx 3 methylation. Conversely, the non-cancerous mucosa with Runx3 methylation may be reversibly brought back to a normal state with the eradication therapy of $H$. pylori.

\section{References}

1. Ito Y and Miyazono K: Runx transcription factors as key targets of TGF-beta superfamily signaling. Curr Opin Genet Dev 13: 43-47, 2003.

2. Li QL, Ito K, Sakakura C, Fukamachi H, Inoue K, Chi XZ, Lee KY, Nomura S, Lee CW, Han SB, Kim HM, Kim WJ, Yamamoto H, Yamashita N, Yano T, Ikeda T, Itohara S, Inazawa J, Abe T, Hagiwara A, Yamagishi H, Ooe A, Kaneda A, Sugimura T, Ushijima T, Bae SC and Ito Y: Causal relationship between the loss of runx3 expression and gastric cancer. Cell 109: 113-124, 2002.

3. Smith MG, Hold GL, Tahara E and El-Omar EM: Cellular and molecular aspects of gastric cancer. World J Gastroenterol 12: 2979-2990, 2006.

4. Fukamachi H, Ito $\mathrm{K}$ and Ito $\mathrm{Y}$ : Runx-/- gastric epithelial cells differentiate into intestinal type cells. BBRC 321: 58-64, 2004.

5. Oshimo Y, Oue N, Mitani Y, Nakayama H, Kitadai Y, Yoshida K, Ito Y, Chayama K and Yasui W: Frequent loss of runx3 expression by promoter hypermethylation in gastric carcinoma. Pathobiology 71: 137-143, 2004.

6. Kim TY, Lee HJ, Hwang KS, Lee M, Kim JW, Bang YJ and Kang GH: Methylation of runx3 in various types of human cancers and premalignant stages of gastric carcinoma. Lab Invest 84: 479-484, 2004.

7. Eslick GD, Lim LL, Byles JE, Xia HH and Talley NJ: Association of Helicobacter pylori infection with gastric carcinoma: a meta-analysis. Am J Gastroenterol 94: 2373-2379, 1999.

8. Tsuji S, Tsuji M, Murata H, Nishida T, Komori M, Yasumaru M, Ishii S, Sasayama Y, Kawano S and Hayashi N: Helicobacter pylori eradication to prevent gastric cancer: Underlying molecular and cellular mechanisms. World J Gastroenterol 12: 1671-1680, 2006.

9. Uemura N, Okamoto S, Yamamoto S, Matsumura N, Yamaguchi S, Yamakido M, Taniyama K, Sasaki N and Schlemper RJ: Helicobacter pylori infection and the development of gastric cancer. N Engl J Med 345: 829-832, 2001.

10. Blaser MJ, Peres-Peres GI, Kleanthous H, Cover TL, Peak RM, Chyou PH, Stemmermann GN and Nomura A: Infection with Helicobacter pylori strains possessing cagA is associated with an increased risk of developing adenocarcinoma of the stomach. Cancer Res 55: 2111-2115, 1995.
11. Maekita T, Nakazawa K, Mihara M, Nakajima T, Yanaoka K, Iguchi M, Arii K, Kaneda A, Tsukamoto T, Tatematsu M, Tamura G, Saito D, Sugimura T, Ichinose M and Ushijima T: High levels of aberrant DNA methylation in Helicobacter pylori infected gastric mucosae and its possible association with gastric cancer risk. Clin Cancer Res 12: 989-995, 2006.

12. Yamazaki S, Kato S, Matsukura N, Ohtani M, Ito Y, Suto H, Yamazaki Y, Yamakawa A, Tokudome S, Higashi H, Hatakeyama M and Azuma T: Identification of Helicobacter pylori and the cag A genotype in gastric biopsies using highly sensitive real-time PCR as a new diagnostic tool. FEMS 44: 261-268, 2005.

13. Japanese research Society for Gastric Cancer Japanese Classification of Gastric Carcinoma (1st English edition) Kanehara, Tokyo, 1995.

14. Koga Y, Kitajima Y, Miyoshi A, Sato K, Sato S and Miyazaki K: The significance of aberrant CHFR methylation for clinical response to microtubule inhibitors in gastric cancer. J Gastroenterol 41: 133-139, 2006.

15. Kang GH, Lee S, Lee HJ and Hwang KS: Aberrant CpG island hypermethylation of multiple genes in prostate cancer and prognostic imtraepithelial neoplasia. J Pathol 202: 233-240, 2004.

16. So K, Tamura G, Honda T, Homma N, Waki T, Togawa N, Nishizuka S and Motoyama T: Multiple tumor suppressor genes are increasingly methylated with age in non-neoplastic gastric epithelia. Cancer Sci 97: 1155-1158, 2006.

17. Kang GH, Lee HJ, Hwang KS, Lee S, Kim JH and Kim JS: Aberrant $\mathrm{CpG}$ island hypermethylation of chronic gastritis, in relation to aging, gender, intestinal metaplasia, and chronic inflammation. Am J Pathol 163: 1551-1556, 2003.

18. Hamilton S and Aaltonen L (ed). Pathology and Genetics. In: Tumours of the Digestive System. WHO Classification of Tumours, ARC Press, 2000.

19. Zsikla V, Hailemariam S, Baumann M, Mund MT, Schaub N, Meier R and Cathomas G: Increased rate of Helicobacter pylori infection detected by PCR in biopsies with chronic gastritis. Am J Surg Pathol 30: 242-248, 2006.

20. Chan A O-O, Lam SK, Wong CY, Wong WM, Yuen MF, Yeung YH, Hui WM, Rashid A and Kwong YL: Promoter methylation of E-cadherin geen in gastric mucosa associated with Helicobacter pylori infection and in gastric cancer. Gut 52: 502-505, 2003.

21. Saadat I, Higashi H, Obuse C, Umeda M, Murata-Kamiya N, Saito Y, Lu H, Ohnishi N, Azuma T, Suzuki A, Ohno S and Hatakeyama M: Helicobacter pylori CagA targets PAR1/MARK kinase to disrupt epithelial cell polarity. Nature 447: 330-333, 2007.

22. Blaser MJ and Atherton JC: Helicobacter pylori persistence: biology and disease. J Clin Invest 113: 321-333, 2004. 